03,09

\title{
Вращение в постоянном электрическом поле сферической частицы при непрерывном освещении, индуцирующем электрический дипольный момент
}

\author{
(C) А.И. Грачев \\ Физико-технический институт им. А.Ф. Иофффе, РАН \\ Санкт-Петербург, Россия \\ E-mail: grach.shuv@mail.ioffe.ru
}

(Поступила в Редакцию 13 октября 2017 г.)

\begin{abstract}
Впервые рассмотрен эффект вращения в постоянном электрическом поле сферической частицы в условиях стационарного освещения, индуцирующего электрический дипольный момент частицы. Кроме общего рассмотрения эффекта представлен анализ возможных механизмов фотогенерации дипольного момента. Приведены оценки таких параметров вращения, как затрачиваемая мощность и угловая скорость. Размер частиц, удовлетворяющих условию получения максимальных значений для указанных параметров, находится в диапазоне $10 \mathrm{~nm}-10 \mu \mathrm{m}$.
\end{abstract}

DOI: $10.21883 /$ FTT.2018.04.45672.284

\section{1. Введение}

Эффект вращения в постоянном электрическом поле диэлектрических частиц, диспергированных в полуизолирующую жидкость, был открыт еще в XIX веке G. Quincke [1] и носит его имя, „вращение Квинке“ (ВК) [2]. Рост интереса к данному явлению, наблюдаемый в последние десятилетия, обусловлен двумя причинами. Во-первых, проявлением ВК при электрореологическом эффекте (отрицательном или положительном), заключающемся в снижении или, соответственно, росте вязкости суспензий при приложении электрического поля [3-5]. Вторая причина - это попытка использования ВК для создания электростатических микромоторов [6,7], например, для MEMS-устройств. Препятствием служат особенности ВК: (i) пороговый, по отношению к величине приложенного поля, механизм вращения и (ii) случайный характер направления оси вращения частиц (при ортогональности направлению поля). При этом роль случайных факторов неизбежно должна возрастать по мере уменьшения размеров частиц. Возможно, по этой причине область размеров ниже $10 \mu \mathrm{m}$ остается пока недоступной для ВК-микромоторов.

В настоящей работе впервые, насколько нам известно, рассматривается явление вращения в постоянном (однородном) электрическом поле сферической частицы, в которой, в условиях постоянного освещения, непрерывно генерируется электрический дипольный момент, ортогональный направлению силовых линий поля. Представлено общее описание эффекта (безотносительно к конкретной физической природе механизма фотогенерации дипольного момента) в условиях реализации так называемого адиабатического вращения. Анализируется механизм генерации электрического дипольного момента в случае трех возможных фотоэффектов, удовлетворяющих симметрии данного явления. Представлены оцен- ки мощности и скорости вращения, а также диапазона оптимальных размеров частиц.

\section{2. Общее рассмотрение}

При теоретическом описании ВК обычно рассматриваются частицы сферической формы, что предполагается и в нашем случае. Общая геометрия эксперимента представлена на рисунке. Сфера фотопроводящего материала радиуса $R$ помещена в диэлектрическую

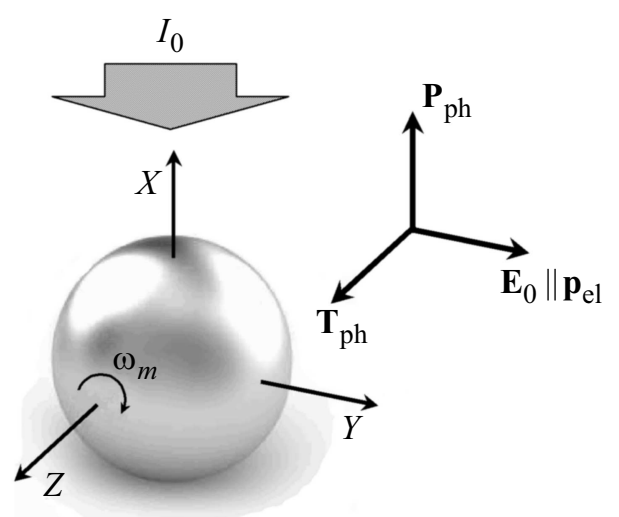

Геометрия эксперимента по фотоиндуцированному вращению сферической частицы радиуса $R$, погруженной в изолирующую жидкость, находящуюся в постоянном однородном электрическом поле $\mathbf{E}_{0}$. Сфера освещается пучком света (интенсивностью $I_{0}$ ), генерирующим в неподвижной частице электрический дипольный момент $\mathbf{p}_{\mathrm{ph}} \perp \mathbf{E}_{0}$, а электрическое поле индуцирует дипольный момент $\mathbf{p}_{\mathrm{el}} \| \mathbf{E}_{0}$. В результате воздействия на сферу вращательного момента $\mathbf{T}_{\mathrm{ph}}$ возникает ее вращение с угловой скоростью $\omega_{m}$. В условиях адиабатического вращения величина и направление индуцируемых дипольных моментов практически совпадают с $\mathbf{p}_{\mathrm{ph}}$ и $\mathbf{p}_{\mathrm{el}}$. 
жидкость, находящуюся в однородном электрическом поле $\mathbf{E}_{0}$. На сферу падает пучок света интенсивности $I_{0}$, распространяющийся вдоль оси $Y$, который в случае неподвижной сферы генерирует в том же направлении дипольный момент $\mathbf{p}_{\mathrm{ph}}$. Взаимодействие электрического поля и диполя индуцирует момент вращения $\mathbf{T}_{\mathrm{ph}}$

$$
\mathbf{T}_{\mathrm{ph}}=\mathbf{p}_{\mathrm{ph}} \times \mathbf{E}_{0},
$$

направленный вдоль оси $Z$ (ось вращения). При обеспечении постоянства величины $\mathbf{T}_{\text {ph }}$ должно наблюдаться стационарное вращение сферы с угловой скоростью $\omega_{m}$, которая определяется равенством между $\mathbf{T}_{\mathrm{ph}}$ и моментом $\mathbf{T}_{l}$, воздействующим на вращающуюся сферу со стороны вязкой жидкости

$$
T_{\mathrm{ph}}=T_{l}=8 \pi \eta R^{3} \omega_{m},
$$

где $\eta$ - динамическая вязкость жидкости.

Ключевым моментом является обеспечение таких значений (фото)проводимости, при которых величина и направление фотогенерируемого дипольного момента

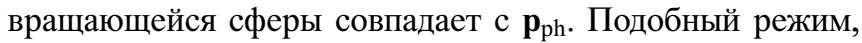
который мы обозначим, как адиабатическое вращение, очевидно, возможен при том условии, что процессы генерации и релаксации фотодиполя могут рассматриваться как мгновенные, в масштабе времен определяемых величиной $\omega_{m}^{-1}$. Рассмотренные ниже механизмы фотогенерации $\mathbf{p}_{\text {ph }}$ связаны с установлением дрейфодиффузионного равновесия внутри освещенной сферы в условиях ее вращения. При этом следует учесть специфику сферической геометрии, что, в частности, выражается в неэкспоненциальном характере установления равновесия. Однако в дальнейшем используется допущение о (квази)экспонециальной зависимости процессов генерации/релаксации заряда с характерным временем $\tau_{\mathrm{g}, \mathrm{rel}}=\tau$. В этом случае условие адиабатического вращения можно выразить следующим неравенством:

$$
\omega_{m} \tau \ll 1
$$

При нарушении условия (3) описание процесса вращения требует введения понятия эффективного дипольного момента, отличного от $\mathbf{p}_{\text {ph }}$, как по величине, так и по направлению, подобно тому, как это делается в случае ВК [2]. Результаты анализа ВК могут быть востребованы в нашем случае, поскольку приложенное поле за счет (фото)проводимости сферы индуцирует дипольный момент $\mathbf{p}_{\mathrm{el}}$, способный повлиять на ее вращение. Поэтому условие адиабатического вращения (3), в том случае, когда $p_{\mathrm{ph}}<p_{\mathrm{el}}$, должно быть усилено

$$
\omega_{m} \tau \ll p_{\mathrm{ph}} / p_{\mathrm{el}},
$$

при этом, учитывая вышесказанное, значения $\tau$ для $p_{\mathrm{ph}}$ и $p_{\mathrm{el}}$ совпадают. Ясно, что условия адиабатического вращения нетрудно реализовать в эксперименте путем задания величины $E_{0}$ и/или $I_{0}$.
Условия реализации ВК и фотоиндуцированного вращения, если говорить о соотношении между проводимостью сферы и окружающей среды, прямо противоположны. Поэтому одновременное проявление этих эффектов, по крайней мере в условиях стационарного освещения, маловероятно. Более того, ВК принципиально является неадиабатическим вращением, поскольку вращающий момент является результатом неколлинеарности электрического поля и эффективного дипольного момента, возникающей в процессе вращения сферы.

\section{3. Механизмы фотогенерации дипольного момента}

Сферическая симметрия обсуждаемого явления заставляет обратиться к фотоэффектам, отвечающим симметрии скаляра или псевдоскаляра. В качестве таковых ниже рассмотрены фотодиффузионный (ФДЭ) и циркулярный фотогальванический эффект (ЦФГЭ) в кристаллах симметрии $T$ и $O$ [8], а также фотовольтаический эффект (ФВЭ). ФДЭ рассматривается в скалярном приближении, учитывая незначительность или полное отсутствие анизотропии поглощения света в кристаллах не слишком низкой симметрии. Полярная симметрия ФВ-эффекта не исключает, тем не менее, возможность его использования, поскольку при сферической форме образца и однородности свойств барьера по его поверхности он также будет удовлетворять требуемому условию симметрии.

Представленный ниже анализ указанных механизмов справедлив в рамках общего допущения, обусловленного спецификой взаимодействия сферы с падающим световым пучком, заключающегося в том, что показатели преломления сферы и диэлектрической жидкости полагаются практически равными.

Фотодиффузионный эффект. Хорошо известно, что при пространственно неоднородной фотогенерации в образце носителей обоих типов (электронов и дырок) внутри освещаемой области возникает стационарное электрическое поле $E_{D}$ (поле амбиполярной диффузии). В том случае, когда толщина образца $d$ в направлении распространения света удовлетворяет условию $\alpha d \ll 1$, где $\alpha-$ коэффициент поглощения света, $E_{D}$ однородно по толщине образца и имеет простой вид

$$
E_{D}=\alpha(k T / e)\left(\mu_{n} \tau_{n}-\mu_{p} \tau_{p} / \mu_{m} \tau_{n}+\mu_{p} \tau_{p}\right) .
$$

Выражение (5) справедливо при предположении о линейной рекомбинации носителей заряда и выполнении условий: $n_{0} \gg n_{d}, p_{0} \gg p_{d}$, где $n_{0}, p_{0}-$ концентрация фотоэлектронов в зоне проводимости и фотодырок в валентной зоне на освещаемой поверхности образца, $n_{d}$ и $p_{d}-$ темновые концентрации соответствующих носителей заряда, $\mu_{n} n, \mu_{p}$ и $\tau_{n}, \tau_{p}-$ подвижность и время жизни электронов и дырок соответственно, $k$ - постоянная Больцмана, $T$ - температура образца, $e$ - заряд электрона. В условиях монополярного 
возбуждения основных носителей заряда выражение (5) еще более упрощается

$$
E_{D}=\alpha(k T / e)\left(n_{0} / n_{d}+n_{0}\right) .
$$

С учетом однородности поля $E_{D}$ внутри сферы величина связанного с ним дипольного момента определяется известным выражением

$$
p_{\mathrm{ph}}=4 \pi \varepsilon_{s} \varepsilon_{0} R^{3} E_{D},
$$

где $\varepsilon_{s}$ - относительная диэлектрическая проницаемость материала сферы, $\varepsilon_{0}$ - диэлектрическая постоянная вакуума.

Максимальная величина $p_{\text {ph }}$ при монополярном возбуждении (оптические переходы с локальных уровней в запрещенной зоне) достигается при значениях $\alpha \leq 10^{4} \mathrm{~cm}^{-1}$, которым отвечает $E_{D} \sim 2.5 \cdot 10^{2} \mathrm{~V} \cdot \mathrm{cm}^{-1}$ (при $\left.n_{d} \ll n_{0}\right)$. Для межзонных переходов $\alpha \geq 10^{5} \mathrm{~cm}^{-1}$, однако, выигрыш может компенсировать противоположное направление диффузионных потоков электронов и дырок. Соотношение между ними может варьироваться в очень широких пределах, поэтому разумным выглядит предположение, что в данном случае $E_{D} \sim 10^{3} \mathrm{~V} \cdot \mathrm{cm}^{-1}$.

Как следует из (5)-(6), при достижении условия $n_{d}, p_{d} \ll n_{0}, p_{0} E_{D}$ практически не зависит от $I_{0}$. Учитывая предполагаемый (квази)экспоненциальный характер установления $E_{D}$, при котором значение $\tau$ близко к максвелловскому времени релаксации, определяемому фотопроводимостью сферы, имеем $\omega_{m} \tau \sim E_{0} / I_{0}$.

Циркулярный фотогальванический эффект. При очевидном различии природы ЦФГЭ $[8,9]$ и ФДЭ феноменологическое описание генерации электрического поля $\left(E_{\mathrm{CPG}}\right)$ практически одинаково. В случае пространственно однородной монополярной генерации (электронов) выражение для $E_{\mathrm{CPG}}$ (при условии $\left.n_{d} \ll n_{0}\right)$ имеет следующий вид:

$$
E_{\mathrm{CPG}}=G / e \mu_{n} \tau_{n}
$$

При этом $J_{\mathrm{CPG}}=\alpha G_{c} I-$ величина генерируемого ЦФГЭ-тока, где $G_{c}$ - так называемый коэффициент Гласса [8] (величина квантового выхода, деленная на энергию фотона, принята равной единице). В общем случае $G_{c}$ является псевдотензором второго ранга, но в кристаллах симметрии $T$ и $O$ сводится к псевдоскаляру. На сегодняшний день можно сослаться лишь на данные исследования ЦФГЭ в кристаллах со структурой силленита (симметрия $T$ ) $\mathrm{Bi}_{12} M \mathrm{O}_{20}(M=\mathrm{Ge}, \mathrm{Si}, \mathrm{Ti})[10,11]$, на которые мы будем далее опираться.

Дипольный момент, генерируемый в случае ЦФГЭ, также определяется выражением (7). По имеющимся оценкам [10] $E_{\mathrm{CPG}} \sim 10^{-2} \mathrm{~V} \cdot \mathrm{cm}^{-1}$, что несопоставимо мало по сравнению с $E_{D}$, однако имеются пути его увеличения. Во-первых, это использование более коротковолнового облучения, отвечающего области начала межзонных переходов, где наблюдается резкий рост удельного оптического вращения [12] и, по-видимому, $G_{c}[11]$.
Второй, возможно более эффективный способ, - это целенаправленное ухудшение фотопроводящих свойств. С учетом высокого значения диэлектрической проницаемости $\mathrm{Bi}_{12} \mathrm{SiO}_{20}\left(\varepsilon_{s}=56\right)$ и относительно низкой подвижности электронов $\left(\mu_{n} \sim 5 \mathrm{~cm}^{2} \mathrm{~V}^{-1} \mathrm{~s}^{-1}\right.$ [13]) сопоставимые с ФДЭ значения $p_{\mathrm{ph}}$ потребуют снижения $\tau_{n}$ примерно до $\sim 10^{-10} \mathrm{~s}$. С учетом величины полей, генерируемых за счет линейного фотогальванического эффекта в кристаллах $\mathrm{LiNbO}_{3}[8]$, задача увеличения ECPG не представляется невыполнимой.

Фотовольтаический эффект. При традиционном использовании ФВЭ возникающий дипольный момент обусловлен краевым эффектом и относительно мал. Это справедливо и в случае одностороннего освещения образца сферической формы, но лишь при условии, что ширина области пространственного заряда барьера $W$ много меньше радиуса сферы. В противном случае, когда $W=R-r$ и $R \gg r$, где $r$ - радиус сферы, отвечающей нейтральной области, распределение заряда, образующегося в результате разделения фотовозбуждаемых электрон-дырочных пар, можно представить как равномерно заряженную полусферу с общим зарядом $Q$ и равного по величине, но противоположного по знаку, точечного заряда в центре сферы. Однако реализация указанной картины требует выполнения еще двух условий: (i) $\alpha W \gg 1$ и (ii) диффузионные длины электронов и дырок много меньше $R$. Простой расчет показывает, что дипольный момент, отвечающий указанному распределению заряда, равен $Q R / 2$. Величина $Q$ достигает своего максимального значения, когда фотоэдс $V_{\mathrm{ph}}$ приближается к величине диффузионной разности потенциалов (барьер Шоттки) или контактной разности потенциалов $p$ - и $n$-областей ( $p-n$ переход). Используя известное выражение для емкости (полу)сферического конденсатора, можно оценить величину $Q$, соответственно, $p_{\mathrm{ph}}$

$$
p_{\mathrm{ph}}=\pi \varepsilon_{s} \varepsilon_{0} R^{3} E_{\mathrm{ph}}(r / R),
$$

где $E_{\mathrm{ph}}=V_{\mathrm{ph}} / W \approx V_{\mathrm{ph}} / R$. Теперь, используя $\alpha \sim 10^{5} \mathrm{~cm}^{-1}$ и предполагая, что для выполнения условия сильного поглощения света достаточно $W \sim 3 \cdot 10^{-5} \mathrm{~cm}$, получим оценку $E_{\mathrm{ph}} \sim 10^{4} \mathrm{~V} \cdot \mathrm{cm}^{-1}$ при $V_{\mathrm{ph}}=0.3 \mathrm{~V}$. Однако в (9) в отличие от (7) входит отношение $(r / R) \leq 0.1$, а также отсутствует четверка, поэтому при сравнении ФВЭ механизма с остальными величина $E_{\text {ph }}$ должна быть уменьшена примерно в сорок раз. Необходимо также отметить одно принципиальное отличие данного механизма, заключающееся в том, что центр плеча диполя будет находиться вне центра сферы.

\section{4. Обсуждение}

Сравнение представленных механизмов показывает, что на данный момент использование ФДЭ выглядит более предпочтительным. Однако следует учесть, что выше мы пренебрегли влиянием поверхностной рекомбинации, в то время как ее роль может быть весьма суще- 
ственной. Так, в пределе бесконечно высокой скорости поверхностной рекомбинации величина (суммарного) дипольного момента должна упасть практически до нуля. Следует подчеркнуть, что в случае ЦФГЭ поверхностная рекомбинация может сыграть скорее положительную роль, способствуя требуемому снижению $\tau_{n}$.

Оценку параметров, важных с точки зрения потенциального использования обсуждаемого явления, начнем с величины мощности, затрачиваемой на вращение сферы. Используя соотношения (1), (2) и (7), получаем

$$
P=T_{\mathrm{ph}} \omega_{m}=T_{\mathrm{ph}}^{2} / 8 \pi \eta R^{3}=2 \pi R^{3}\left(\varepsilon_{s} \varepsilon_{0} E_{0} E_{D}\right)^{2} / \eta,
$$

а для удельной мощности $U$ (отнесенной к объему сферы)

$$
U=(3 / 2 \eta)\left(\varepsilon_{s} \varepsilon_{0} E_{0} E_{D}\right)^{2} .
$$

Предполагая, что $E_{0}=10^{4} \mathrm{~V} \cdot \mathrm{cm}^{-1}$ (характерное для ВК значение порогового поля [2]) и $E_{D}=10^{3} \mathrm{~V} \cdot \mathrm{cm}^{-1}$ для $\varepsilon_{s} \sim 10$ и $\eta \sim 10^{-2} \mathrm{~Pa} \cdot \mathrm{s}$ (трансформаторное масло), получаем $U \sim 10^{-2} \mathrm{pW}_{\mu \mathrm{m}^{-3}}$ и $\omega_{m} \sim 5 \cdot 10^{2} \mathrm{~s}^{-1}$.

Ограничения на условия поглощения света определяют диапазон размеров сферы, оптимальных для того или иного механизма. Для ФДЭ и ЦФГЭ нижней границы в принципе нет, она будут определяться, например, технологическими условиями или влиянием квантово-размерных эффектов. Верхняя граница лежит в области $R \sim 0.1 \mu \mathrm{m}$, а использование монополярного возбуждении для ФДЭ может сдвинуть ее на одиндва порядка (при сопутствующем снижении величины $\left.p_{\mathrm{ph}}\right)$. Для ФВЭ-механизма существует нижняя граница $R \sim 0.3 \mu \mathrm{m}$, верхняя примерно на порядок выше (использование области начала межзонных переходов). Таким образом, вполне разумным выглядит реализация широкого диапазона размеров от $10 \mathrm{~nm}$ до $10 \mu \mathrm{m}$.

Форма вращающегося объекта может отличаться от сферической при условии сохранения величины и направления $\mathbf{p}_{\text {ph }}$ при повороте вокруг оси вращения на любой угол. Оно выполняется, например, для частиц, имеющих форму цилиндра, при соответствующей ориентации относительно направления поля. Более того, если фотоактивные сферические частицы заполняют или впрессованы в объект цилиндрической формы, то он также испытает вращение. Подобный вариант конструкции микро(нано)моторов может оказаться более привлекательным с технологической точки зрения.

В заключении укажем на недавно опубликованную работу по оптически индуцированному изменению вязкости суспензии, содержащей мелкодисперсный $(25-140 \mathrm{~nm})$ порошок $\mathrm{TiO}_{2}$ [14]. Авторы связывают наблюдавшийся рост вязкости с индуцируемым светом дипольным моментом частиц. Однако отсутствие выделенного полярного направления в эксперименте ставит под сомнение предложенное авторами объяснение [14], если не предположить, что причиной появления у частиц дипольного момента является фотодифузионный эффект.

\section{5. Заключение}

В настоящей работе впервые рассмотрено явление вращения в постоянном (однородном) электрическом поле сферической частицы, помещенной в диэлектрическую жидкость, в которой при ее постоянном освещении непрерывно генерируется электрический дипольный момент, ортогональный направлению силовых линий поля. Дано общее описание эффекта в условиях реализации так называемого адиабатического вращения. Анализ трех возможных механизмов фотогенерации дипольного момента (удовлетворяющих симметрии данного явления), - фотодифузионного, фотовольтаического и за счет циркулярного фотогальванического эффекта, показал, что на сегодняшний день первый из них выглядит наиболее предпочтительным. Представлены оценки мощности и скорости вращения при реализации фотодиффузионного механизма. Результаты работы позволяют предположить, что явление фотоиндуцированного вращения частиц в постоянном электрическом поле может стать базой для создания микро(нано)моторов с характерными размерами, перекрывающими область от $10 \mathrm{~nm}$ до $10 \mu \mathrm{m}$.

Автор выражает благодарность А.А. Камшилину за полезное обсуждение работы.

\section{Список литературы}

[1] G. Quincke. Ann. Phys. Chem., Band 59, 417 (1896).

[2] T.B. Jones. Electromechanics of Particles. Cambridge University Press, Cambridge, N.Y. (1995).

[3] Yu. Dolinsky, T. Elperin. Phys. Rev. E 85, 026608 (2012).

[4] D. Das, D. Saintillan. Phys.Rev. E 87, 043014 (2013).

[5] N. Pannacci, E. Lemaire, L. Lobry. Rheol. Acta 46, 899 (2007).

[6] A. Jakli, B. Senyuk, G. Liao, O.D. Lavrentovich. Soft Matter 4, 2471 (2008).

[7] R.A. Bauer, L. Kelemen, M. Nakano, M. Totsuka, M. Zrinyi. Smart Mater. Struct. 24, 105110 (2015).

[8] Б.И. Стурман, В.М. Фридкин. Фотогальванический эффект в средах без центра симметрии. Наука, М. (1992).

[9] E.L. Ivchenko, G.E. Pikus. Superlattices and Other Heterostructures: Symmetry and Optical Phenomena. SpringerVerlag, Berlin (1995).

[10] М.П. Петров, А.И. Грачев. Письма ЖЭТФ 30, 18 (1979).

[11] М.П. Петров, А.И. Грачев. ФТТ 22, 1671 (1980).

[12] В.А. Кизель, В.И. Бурков, Ю.И. Красилов, Н.Л. Козлова, Г.М. Сафронов, В.Н. Батог. Оптика и спектроскопия 34, 1165 (1973).

[13] S.I. Sochava, K. Buse, E. Krätzig. Phys. Rev. B 51, 4684 (1995).

[14] Y.R. Smith, D. Heermance, R.N. Smith. Korea-Australia Rheology J. 28, 51 (2016). 